\title{
Intracranial Aneurysm Segmentation in 3D CT Angiography: Method and Quantitative Validation
}

\author{
Azadeh Firouzian $^{* a, b}$, R. Manniesing ${ }^{\mathrm{a}, \mathrm{b}}$, Z.H. Flach ${ }^{\mathrm{b}}$, R. Risselada ${ }^{\mathrm{a}}$, F. van Kooten ${ }^{\mathrm{c}}$, M.C.J.M. \\ Sturkenboom ${ }^{\mathrm{a}, \mathrm{d}}$, A. van der Lugt ${ }^{\mathrm{b}}$, W.J. Niessen ${ }^{\mathrm{a}, \mathrm{b}, \mathrm{e}}$ \\ ${ }^{a}$ Department of Medical Informatics, Erasmus MC, University Medical Centre Rotterdam, the \\ Netherlands \\ ${ }^{\mathrm{b}}$ Department of Radiology, Erasmus MC, University Medical Centre Rotterdam, the Netherlands \\ ${ }^{\mathrm{c}}$ Department of Neurology, Erasmus MC, University Medical Centre Rotterdam, the Netherlands \\ ${ }^{\mathrm{d}}$ Department of Epidemiology, Erasmus MC, University Medical Centre Rotterdam, the Netherlands \\ ${ }^{\mathrm{e}}$ Department of Imaging Science and Technology, Faculty of Applied Sciences, Delft University of \\ Technology, the Netherlands
}

\begin{abstract}
Accurately quantifying aneurysm shape parameters is of clinical importance, as it is an important factor in choosing the right treatment modality (i.e. coiling or clipping), in predicting rupture risk and operative risk and for pre-surgical planning. The first step in aneurysm quantification is to segment it from other structures that are present in the image. As manual segmentation is a tedious procedure and prone to inter- and intra-observer variability, there is a need for an automated method which is accurate and reproducible. In this paper a novel semi-automated method for segmenting aneurysms in Computed Tomography Angiography (CTA) data based on Geodesic Active Contours is presented and quantitatively evaluated. Three different image features are used to steer the level set to the boundary of the aneurysm, namely intensity, gradient magnitude and variance in intensity. The method requires minimum user interaction, i.e. clicking a single seed point inside the aneurysm which is used to estimate the vessel intensity distribution and to initialize the level set. The results show that the developed method is reproducible, and performs in the range of interobserver variability in terms of accuracy.
\end{abstract}

Keywords: intracranial aneurysm, GAC, level set, segmentation, evaluation

\section{INTRODUCTION}

Intracranial aneurysms are pathological dilations of the major arteries crossing through the subarachnoid space at the base of the brain. They often occur near the bifurcations of Circle of Willis (Figure 1) including anterior communicating (ACoA), posterior communicating ( $\mathrm{PCoA}$ ), middle cerebral (MCA), internal carotid (ICA) and basilar artery. Unruptured brain aneurysms do not have indicative symptoms associated and are therefore difficult to diagnose before rupture. The prevalence of brain aneurysm is estimated $2-3 \%$ in the general population. Incidentally found aneurysms tend to have lower risk of rupture than aneurysms found additional to a ruptured aneurysm. The subarachnoid hemorrhage (SAH) resulting from the rupture is a devastating event and is associated with a high mortality and morbidity rate. It can be prevented if the aneurysm is treated before rupture [1],[2]. Multiple factors can have an effect on the rupture risk of the aneurysm such as environmental factors (e.g. smoking, hypertension and alcohol consumption) [1], size [3], [4], [5], [6], [7] and shape [8], [9], [10].

Next to aneurysm volume, aneurysm shape characterization has recently received considerable attention [10]. Automated assessment of aneurysm volume and shape has the potential to improve diagnostic accuracy, to reduce operator time and to allow objective assessment of aneurysm shape changes in longitudinal studies of unruptured aneurysms. Also it can contribute for making models for in computational fluid dynamics (CFD) simulations which leads to better planning in

*a.firouzian@erasmumc.nl ; phone 3110 7044124; fax 31107044722 ; www.bigr.nl

Medical Imaging 2010: Image Processing, edited by Benoit M. Dawant, David R. Haynor,

Proc. of SPIE Vol. 7623, 76233M - @ 2010 SPIE · CCC code: 1605-7422/10/\$18 · doi: 10.1117/12.843674 
minimally invasive surgeries. Both for volume assessment and shape characterization, aneurysm segmentation is required, for which only a few methods have been described in literature.

Law et al. developed a method based on multi-range filters and local variances to perform segmentation of intracranial aneurysms on Phase Contrast Magnetic Resonance Angiography (PCMRA) data [11]. Hernandez et al. presented a method for intracranial aneurysm segmentation based on geometric deformable models and applied them to CTA and 3D Rotational Angiography (3DRA) data [12]. In this work they developed a method based on Geometric Active Regions (GAR) which requires training on a number of training data to get statistical information on the intensity.

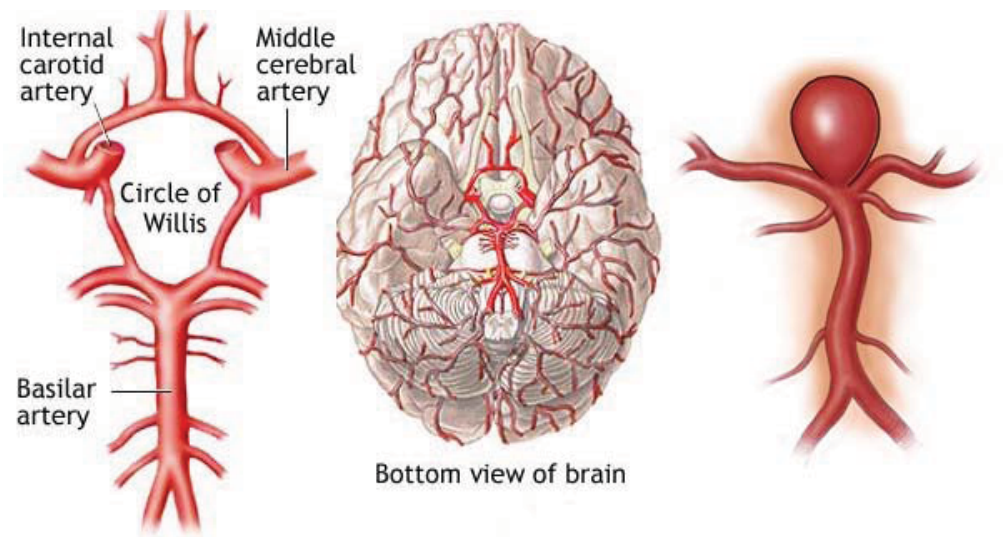

Figure 1. Schematic of an aneurysm located on top of the basilar artery (right) and Circle of Willis (left) [13]

In this paper, a semi-automatic aneurysm segmentation method based on Geodesic Active Contours (GAC) for segmenting brain aneurysms from 3D CTA is presented and quantitatively evaluated. The method is implemented in the level set frame work. Three features namely; intensity, gradient and intensity variance are extracted form the image and integrated in the speed function in a way that the level set evolution is steered towards the aneurysm boundaries. To our knowledge this is the first work in which a method has been presented for cerebral aneurysm segmentation in CTA and which has been evaluated using manually obtained segmentations by expert observers.

\section{METHOD}

Our intracranial aneurysm segmentation method is based on GAC, introduced by Casselles et al. [14], [15]. In this framework the evolving segmentation of the 3D aneurysm wall is represented by the zero level set of an embedding function. By determining the way in which the embedding function evolves, the $3 \mathrm{D}$ surface representing the segmentation evolves towards the aneurysm boundaries. The evolution of the embedding function $\Phi$ is described by the following Partial Differential Equation (PDE):

$$
\Phi_{t}-\mu \nabla F \nabla \Phi+F\left(1-\varepsilon k_{\text {mean }}\right)|\nabla \Phi|=0
$$

where $\mathrm{F}$ is the speed function, which drives the the zero level set towards the aneurysm wall, $\varepsilon$ is a weighting factor of a (curvature-based) smoothing term, $\mathrm{k}_{\text {mean }}$ is the mean curvature and $\mu$ is the weighting factor of the advection term. For aneurysm segmentation $\mathrm{k}_{\text {mean }}$ has been chosen as aneurysms generally have a spherical shape and therefore smoothness is desired in all directions. The level set is initialized by a surface with fixed radius with origin at the user-selected seed point and after convergence of Equation (1); the zero level set defines the aneurysm boundaries. In order to initialize the level set, a single seed point is defined by the user [15], [16].

In level set segmentation, the selection of a proper speed function is essential. In our case, the speed function is based on three different image features. The intensity feature $\left(\mathrm{F}_{\mathrm{i}}\right)$ is calculated based on the estimated intensity distributions of the aneurysm $\left(\mathrm{g}_{\mathrm{v}}\right)$ and background $\left(\mathrm{g}_{\mathrm{b}}\right)$ which are modeled by a normal distribution [17]. $\mathrm{g}_{\mathrm{v}}$ is estimated by calculating the intensity statistics in a neighborhood around the single seed point inside the aneurysm and $\mathrm{g}_{\mathrm{b}}$ by thresholding the image in the interval of $[-200,200] \mathrm{HU}$. Then the intensity speed image is defined as follows: 


$$
F_{i}=\frac{g_{v}-g_{b}}{g_{v}+g_{b}}
$$

$\mathrm{F}_{\mathrm{i}}$ will be zero at the intersection of the two Gaussian distributions of the vessels and background and can vary between 1 and -1 for high and low intensity values respectively.
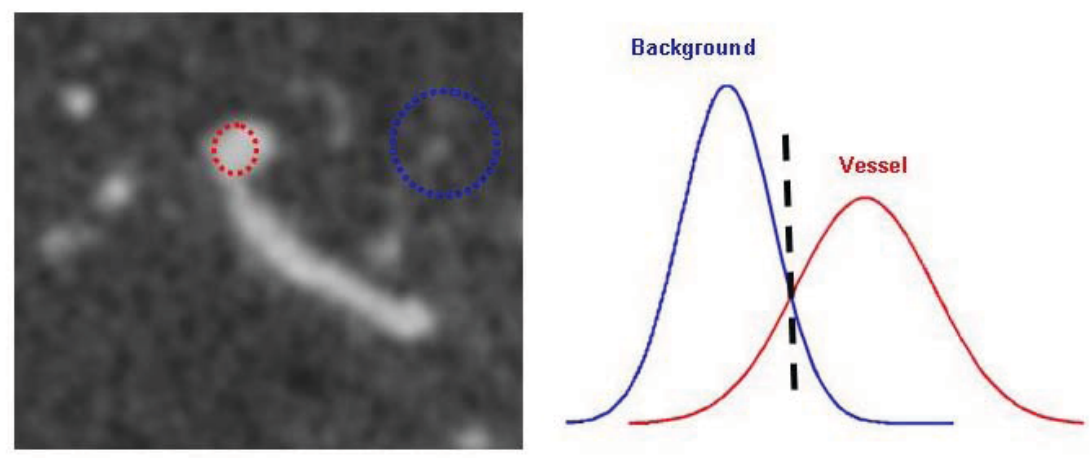

Figure 2. The Gaussian model for estimating intensity values in vessel and background has been shown (right) together with the corresponding neighborhood in an example ROI.

The second image feature is based on the gradient magnitude, and is scaled between 0 and 1 as follows:

$$
F_{\text {grad }}=e^{-\left(\frac{|\nabla I|}{\gamma}\right)^{2}}
$$

where $|\nabla I|$ is the gradient magnitude, and $\gamma$ is a tuning factor. Using this feature we slow down the zero level set near the edges. When there is high gradient in the image, $\mathrm{F}_{\text {grad }}$ will have a lower value and therefore the speed decreases.

Finally, as the intensity inside the vessels in CTA is assumed to be homogeneous, we use the local standard deviation of the intensity to distinguish vessels from other structures. Scaling this speed function also between 0 and 1 , and with the aim to reduce evolution speed in regions with high variance, we choose:

$$
F_{n}=e^{-\left(\frac{\sigma}{\beta}\right)^{2}}
$$

where $\sigma$ is the local standard deviation and $\beta$ is the tuning factor.

The final speed image includes all three components and is defined as follows:

$$
F=F_{i} \cdot F_{\text {grad }} \cdot F_{n}
$$

F ranges between -1 and 1 considering the definition of each individual speed term.

\section{EXPERIMENTS AND RESULTS}

The method has been applied to 15 aneurysms from brain CTA data of 12 patients that were part of a retrospective single-center observational study on subarachnoid hemorrhage. Scans were performed on a Siemens 16-detector row CT scanner (Somatom X-32 Sensation 16; Siemens Medical Solutions, Erlangen, Germany). The scan volume started from the upper limit of the posterior arch of the atlas and extended cephaled with a coverage of $100 \mathrm{~mm}$. Eighty ml contrast material (Iodixanol $320 \mathrm{mg} / \mathrm{ml}$ - Visipaque - Amersham Health, Little Chalfont, UK) was injected using a power injector (EnVision - MedRAD, Pittsburgh, PN, USA) through an 18-20G iv cannula (depending on the size of the vein), in an antecubital vein with an injection rate of $4 \mathrm{ml} / \mathrm{sec}$. For both the anterior and the posterior circulation $1 \mathrm{~mm}$ images with $0.6 \mathrm{~mm}$ overlap were reconstructed with a FOV of $100 \mathrm{~mm}$. 
Three different evaluation measures have been used for this study:

- Similarity Index (SI) which is the amount of overlap between two segmentations:

$$
\mathrm{SI}=2 \frac{\left|S_{\text {Auto }} \cap S_{\text {Man }}\right|}{\left|S_{\text {Auto }}\right|+\left|S_{\text {Man }}\right|}
$$

where $\mathrm{S}_{\text {Auto }}$ represents the automated segmentation and $\mathrm{S}_{\mathrm{Man}}$ represents manual segmentation.

- Average Surface Distance (ASD) which is the average distance between two segmentation surfaces and is approximated as follows:

$$
\mathrm{ASD}=\mathrm{h} / 2 *\left(\mathrm{~V}_{\mathrm{MS}}-\mathrm{V}_{\mathrm{AS}}\right) /\left[\left(\mathrm{D}_{\mathrm{MS}}-\mathrm{V}_{\mathrm{MS}}\right)+\left(\mathrm{V}_{\mathrm{MS}}-\mathrm{E}_{\mathrm{MS}}\right)\right]
$$

Where $V_{M S}$ is the volume of the manual segmentation, $V_{A S}$ is the volume of the automatic segmentation; $D_{M S}$ is the dilated manual segmentation of 1 voxel with a spherical kernel; $\mathrm{E}_{\mathrm{MS}}$ is the eroded manual segmentation of 1 voxel and $\mathrm{h}$ is the voxel size (isotropic).

- Volume Difference (VD) which is the difference in the volume of the aneurysm dome in manual segmentation and the identical volume in the automatic version.

The developed method has been implemented using the GAC module from Insight Toolkit (ITK) [18] which uses a standard forward Euler discretization scheme for the numerical approximation matrix.

The data sets were divided into a training (10 data sets) and a test set (5 data sets). Most of the parameters involved in the segmentation can be fixed: the number of iterations (2000), maximum RMS error which determines the stop criteria of evolution (0.001), radius of the level set at initialization ( 2 pixel sizes; $0.6 \mathrm{~mm}$ ), radius of the neighborhood around the seed point to estimate vessel intensity distribution (2 pixels sizes), scale of gradient (1 pixel size; $0.3 \mathrm{~mm}$ ) and the radius at which the standard deviation is calculated ( 2 pixel sizes). Only three parameters require optimization: the curvature scaling ( $\varepsilon$ in Equation 1$)$ and the tuning parameters for gradient $(\gamma$ in Equation 3$)$ and noise $(\beta$ in Equation 4$)$ speed images. After a pilot study, the curvature scaling which smoothes the final segmentation result was varied in the range of $[0,0.1] \mathrm{mm}^{-1}$, the gradient tuning parameter was varied in the range of $[10,400]$ and noise tuning parameter was varied in the range of $[10,200]$.

To reduce calculation time and memory usage, the data sets were all cropped to a region of interest of $3 \times 3 \times 3 \mathrm{~cm}$ around the aneurysm (utilizing the single seed point within the aneurysm which is required by our method). This region of interest (ROI) was chosen around the user defined seed point.

Two expert radiologists manually segmented all 15 aneurysms for evaluation of the method using a tool in MeVisLab software (Figure 3) [19]. Both observers drew contours around the aneurysm slice by slice by clicking points on the border of the aneurysms. These contours were later converted to a binary mask to create the segmentation.

The manual segmentations of one observer (A) have been chosen to be the ground truth, and were used for training and testing of the method. The manual segmentations of the second observer (B) were also used for testing the method, and to determine the inter-observer variability. Curvature scaling, gradient tuning and noise tuning were optimized on the training set using the three evaluation measures namely, SI, ASD and VD. The optimization was based on selecting the setting which had the best result using the measures.

The manual segmentations performed by the experts only cover the aneurysm whereas the automatic segmentations include the surrounding vessels as well (Figure 4). Therefore in the evaluation procedure, the aneurysm was separated from the surrounding vessels using the manual segmentation (Figure 5). The dilated (by 2 voxels) version of the manual segmentation was used as a mask to divide the segmentation in two parts; one part only including the aneurysm and the other only including the surrounding vessels. The vessel part was dilated with the condition that it is limited to the original segmentation. This is done to compensate for the part of the parent artery which was excluded by the dilated manual mask. The resulting vessel segmentation is then subtracted from the original segmentation to get only the aneurysm part.

The results of training, testing, overall experiments and the inter-observer variability with respect to the three evaluation measures are reported in Table 1. It can be observed that the SI between the automated method and both observer A and 
B is large, and larger than the SI between observers. Also the average surface distance and volume difference between the method and observers is clearly within the range and often smaller than the inter-observer variability. It can also be observed that performance of the method on the training set is similar as on the test set, indicating that the method is not over-trained.
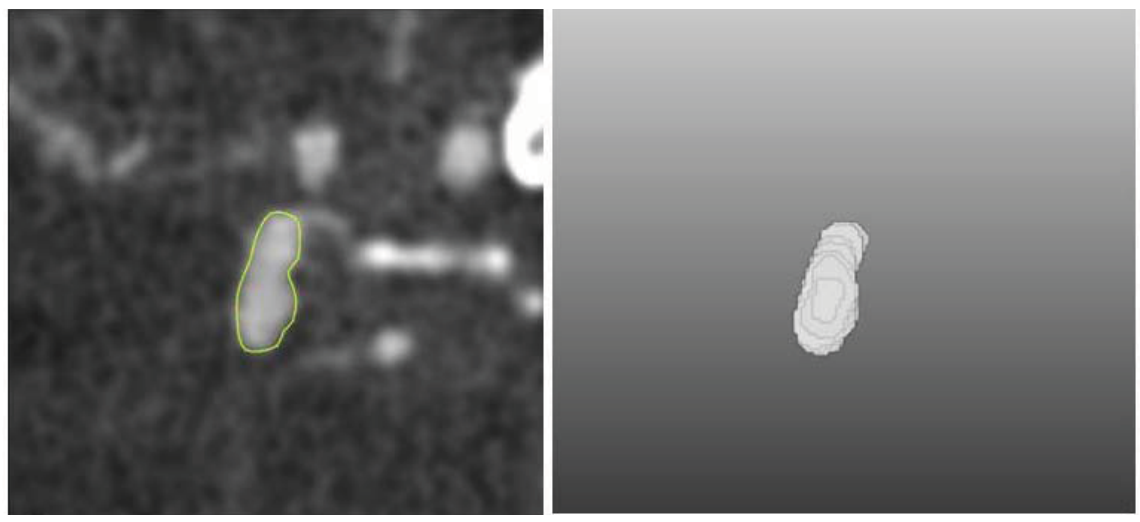

Figure 3. Manually drawn contours around the dome of an aneurysm) on a slice of a 3D CTA data set in an axial view (left); The 3D volume rendering of the binary mask created from the contours which is used in the evaluation section (right). As the contours are made slice by slice, the resulting volume is not smooth.
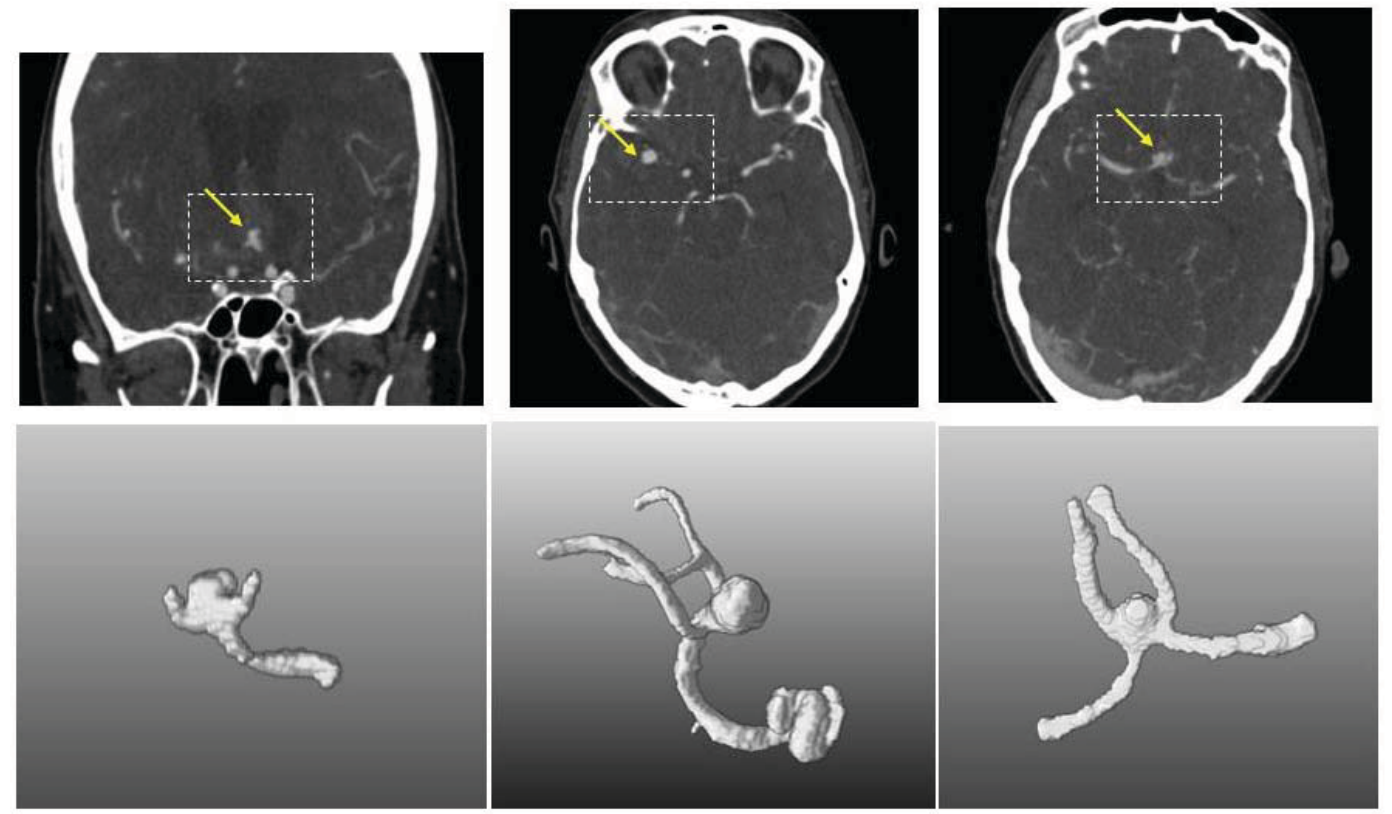

Figure 4. Three examples of segmentation results are shown in this figure. The top row shows the original CTA images on which the ROIs are marked and the bottom row shows the corresponding volume rendered segmentation results. Arrows indicate the location of the aneurysms. From left to right, the aneurysms are located on anterior communicating artery, middle cerebral artery and anterior communicating artery. 

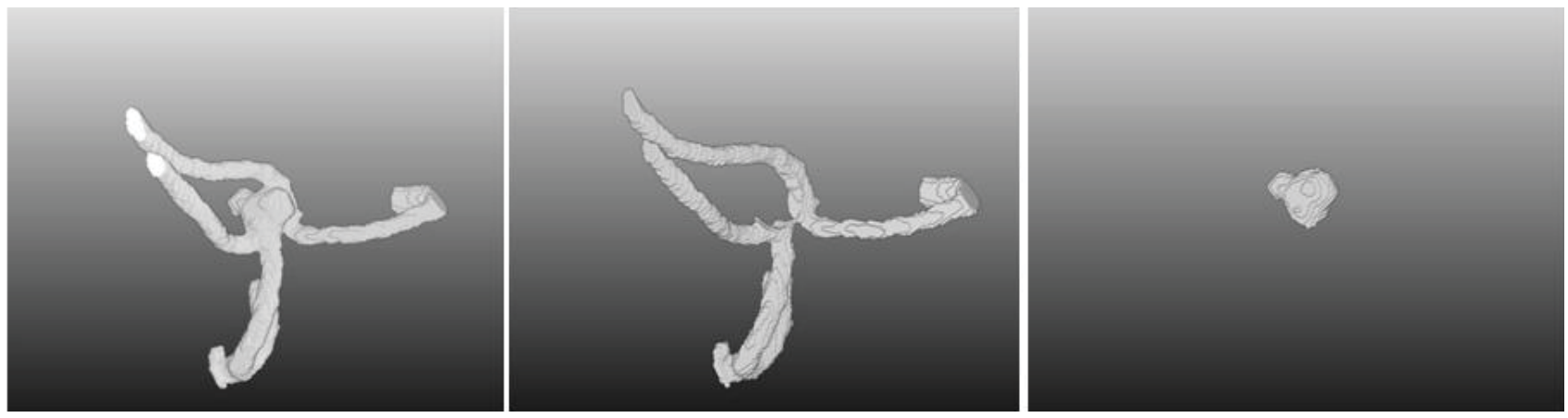

Figure 5. Volume rendering of an automatic segmentation result (left) and aneurysm (right) separated from surrounding vessels (middle) for evaluation purposes. This aneurysm is located on the anterior communicating artery on the left side.

Table 1. Evaluation results of the segmentation method with respect to three different measures. The results are averaged over the data sets. (The average volume of the 15 aneurysms included in the study was $70.1 \mathrm{~mm}^{3}$ )

\begin{tabular}{|c|c|c|c|c|c|c|c|c|c|}
\cline { 2 - 10 } & \multicolumn{2}{|c|}{ Method versus Observer A } & \multicolumn{2}{c|}{ Method versus Observer B } & \multicolumn{4}{c|}{ Inter-observer } \\
\cline { 2 - 10 } & Training & Testing & Overall & Training & Testing & Overall & Training & Testing & Overall \\
\hline SI & 0.838 & 0.851 & 0.842 & 0.83 & 0.81 & 0.82 & 0.731 & 0.781 & 0.747 \\
\hline ASD [mm] & 0.149 & 0.148 & 0.149 & 0.170 & 0.204 & 0.182 & 0.227 & 0.212 & 0.222 \\
\hline VD [mm ${ }^{3}$ ] & 16.142 & 13.459 & 15.248 & 17.759 & 24.471 & 19.996 & 20.938 & 26.519 & 22.799 \\
\hline
\end{tabular}

\section{DISCUSSION AND CONCLUSIONS}

CTA is the most widely used imaging modality for investigating intracranial aneurysms. Performing aneurysm segmentation using CTA data is a challenging task considering the relatively small size of the aneurysms with respect to image resolution and the fact that vessel intensity values overlap with ones from skull base. Having a segmentation of the aneurysm is an essential step towards automatic quantification of aneurysmal volume and shape parameters which in clinical practice may support the pre-operative planning in choosing the right size and type of the first coil.

A novel semi-automatic method for segmentation of brain aneurysms in CTA has been developed. The method performs in the range of the inter-observer variability therefore it is accurate and reproducible and thus has the potential to replace manual segmentation. Also, we hypothesize that in longitudinal studies, the use of automated segmentation is potentially better suited to pick up subtle changes in aneurysm volume and shape. This hypothesis is subject of further research.

There are a couple of limitations in the method that should be addressed. Considering that the focus of the study was on determining the volume of the aneurysm and the method is segmenting both aneurysm and the surrounding vasculature, we had to separate the aneurysm from the vasculature. This was performed using the reference manual annotations. Automatic separation of the aneurysm from the surrounding vasculature would be the best way and recently a method with minimum user interaction has been developed which can be used [20]. The other aspect of our segmentation method which needs more attention is that in cases where the aneurysm or its surrounding vessels are very close to the skull base, the segmentation may leak to the bone area (Figure 6). Using the tool for separation of the aneurysm from the surrounding vessels can also be a solution to this problem. As bone also needs to be removed in this situation, it will be more complicated than the previously mentioned case. 

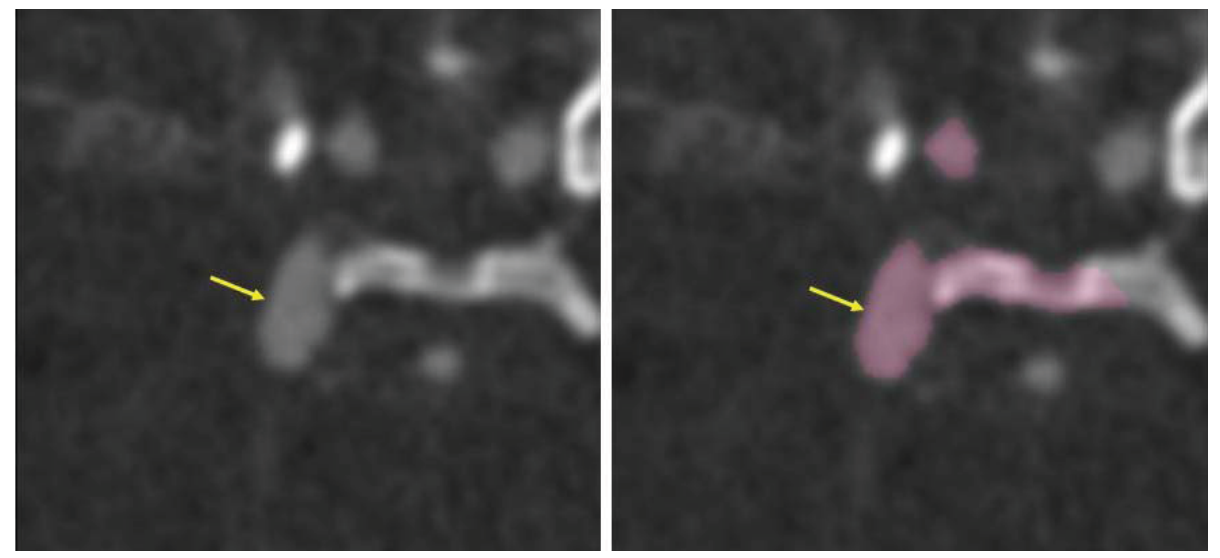

Figure 6. An example of a segmentation result performed on the ROI around the aneurysm. The original CTA image is shown on the left and corresponding segmentation results overlaid on the CTA image on the right. Arrows indicate the location of the aneurysm. A post-processing step is required to separate the aneurysm from both the vasculature and bone. In this case the vasculature adjacent to the aneurysm grew into bone which can cause complications in the evaluation process.

\section{REFERENCES}

[1] Schievink, W.I., "Intracranial Aneurysms," N Engl J Med, 336, $28-40$ (1997).

[2] Beck, J., S. Rohde, J. Berkefeld, V. Seifert, A. Raabe, "Size and location of ruptured and unruptured intracranial aneurysms measured by 3D rotational angiography," Surgical Neurology, 65, 8-27 (2006).

[3] Hademenos, G, Masssoud, T, Turjman, F, et al., "Anatomical and morphological factors correlating with rupture of intracranial aneurysms in patients referred for endovascular treatment," Neuroradiology, 40(11), 755-760 (1988).

[4] Lall RR, Eddleman CS, Bendok BR, et al., "Unruptured intracranial aneurysms and the assessment of rupture risk based on anatomical and morphological factors: sifting through the sands of data," Neurosurg Focus, 26(5), E2 (2009).

[5] Tremmel, M, Dhar, S, Levy, EI, Mocco J, et al., "Influence of intracranial aneurysm-to-parent vessel size ratio on hemodynamics and implication for rupture: results from a virtual experimental study," Neurosurgery, 64(4), 622-630 (2009).

[6] Jeong, YG, Jung, YT, Kim, MS, et al., "Size and location of ruptured intracranial aneurysms," J Korean Neurosurg Soc., 45(1), 11-5 (2009).

[7] Jou, LD, Mawad, ME., "Growth rate and rupture rate of unruptured intracranial aneurysms: a population approach," Biomed Eng Online, 8, 11 (2009).

[8] Millán, R, Dempere-Marco, L, Pozo, J, et al., "Morphological characterization of intracranial aneurysms using 3D moment invariants," IEEE Trans. Med. Imag., 26(9), 1270-1282 (2007).

[9] De Rooij, NK, Velthuis, BK, Algra, A, et al., "Configuration of the Circle of Willis, Direction of flow, and shape of the aneurysm as risk factors for rupture of intracranial aneurysms," J Neurol, 256(1), 45-50 (2009).

[10] Raghavan, M., L., Ma, B., Harbaugh, R., E., "Quantified Aneurysm Shape and Rupture Risk," J Neurosurg, 102, 355-362 (2005).

[11] Law, MWK. , ACS. , Chung, "Vessel and Intracranial Aneurysm Segmentation Using Multi-range Filters and Local Variances,” MICCAI 2007, Part I, LNCS 4791, 866-874 (2007).

[12] Hernandez, M. and Frangi, A.F., "Non-parametric Geodesic Active Regions: Method and Evaluation for Cerebral Aneurysms Segmentation in 3DRA and CTA," Medical Image Analysis, 11, 224-241 (2007).

[13] MedlinePlus, http://www.nlm.nih.gov/medlineplus/ency/imagepages/18009.htm

[14] Caselles, V., R. Kimmel and G. Sapiro, "Geodesic Active Contours," International Journal of Computer Vision, 22(1), 61-79 (1997).

[15] Sethian, JA., "Level set methods and fast marching methods," ISBN 0521645573, Cambridge University Press, (1999).

[16] Sethian, JA., "A fast marching level set method for monotonically advancing fronts," Proceedings of the National Academy of Sciences, 93(4), 1591-1595 (1996). 
[17] Manniesing, R., B.K. Velthuis, M.S. van Leeuwen, I.C. van der Schaaf, P.J. van der Laar and W.J. Niessen, "Level Set Based Cerebral Vasculature Segmentation and Diameter Quantification in CT Angiography," Medical Image Analysis, 10(2), 200-214 (2006).

[18] Insight Toolkit, Version 3.4, http://www.itk.org/

[19] MeVisLab, Version 1.6, Bremen, Germany: MeVis Medical Solutions AG (2008). http://www.mevislab.de/

[20] Ford MD, Hoi Y, Piccinelli M, et al., "An objective approach to digital removal of saccular aneurysms: technique and applications," The British Journal of Radiology, In press (2009). 